\title{
Application of Computer-Based Distance Education with Dynamic Webpages in Financial Management Courses
}

\author{
https://doi.org/10.3991/ijet.v16i14.24047 \\ Fang Yu \\ Shijiazhuang University of Applied Technology, Shijiazhuang, China \\ daimengpao118@163.com
}

\begin{abstract}
Financial management is one of most important type of professional courses in the discipline of business management. The information-based teaching of the courses is a hot spot in the research of professional education. As a cutting-edge information technology, webpage technology provides a good information carrier that enables fast sharing, timely access, and opinion exchange of information. The application of information technology to distance education of financial management could promote the learning efficiency of the students. This paper mainly designs a computer-based distance education system with the aid of dynamic webpages. The system encompasses such modules like database, classroom module, and question and answer (Q\&A) module. By introducing the operating mechanism of the system, this paper fully integrates the teaching resources and practices of financial management into the dynamic webpage-based system, providing students or trainees a convenient way of online learning. Through the integration of information technology in education, the proposed system promotes the reform of traditional education model, helps to improve teaching quality and learning efficiency, and enhances the students' ability to analyze and implement financial management.
\end{abstract}

Keywords-Dynamic webpages, financial management, computer technology, distance education, website

\section{Introduction}

Financial management is a compulsory course for students majoring in economics and business management related disciplines. Nowadays, the theoretical knowledge and professional training of financial management courses are mature enough, however, in order to cultivate excellent financial management technicians and senior professionals, it's quite necessary to further improve the established financial management education system [1-3]. In addition, various aspects of financial management, such as financial decision-making, financial analysis, and financial planning and control, are closely related to the development and value of the enterprise, and financial management is an indispensable quality for enterprise managers [4-7]. The traditional teaching and learning methods are based on the face-to-face lectures given by teachers 
in the classroom, such education mode has great limitations in terms of learning time and ways of communication, therefore, at present, quickly and effectively improving the learning efficiency of professionals and optimizing their learning methods have become important contents in the business management of enterprises.

The development of computer and information technologies has promoted the application of computers and information-based teaching in schools or other education institutions, it gives impetus to the educational reform and teaching process optimization works, and it is quite conductive to propelling the development of quality education and information education [8-10]. Distance education is a teaching mode that transfers professional knowledge to students via multimedia or network and provides them with platforms for communication and exchange [11-13]. It takes sharing resources, ensuring education quality, realizing interactions as the teaching goals, and it offers a learning and exchange platform for people who cannot receive formal education or who want to conduct in-depth learning [14-16].

Moreover, distance education and information-based teaching build a bridge for the communication between teachers and students, and between learners of different levels, realizing mutual assistance between different professional levels and the integration of different disciplines [17-19]. Hence, we can foresee that distance education will play an increasingly important role in education, and become one of the important ways to train professionals for different fields [20, 21]. In terms of financial management, developing and applying computer-based distance education system for financial management courses are urgent needs of the business management education.

To this end, this study attempts to design a computer-based distance education system with the aid of dynamic webpages, multimedia technologies, and coding design; the proposed system aims to provide students with a distance learning and exchange platform of financial management courses, and offer teachers a convenient networkaided teaching method; it develops an online Q\&A and exchange space, which can free users from the limitations of offline class time. With the proposed system/website, learners could browse webpages with dynamic and interactive features, the webpages are no longer simplex, which can enhance learners' interest in learning. This paper is of great significance for enhancing the professional ability of learners in financial management and promoting the development of intelligent education.

\section{Webpage Developing Technologies}

\subsection{Web technology}

Computer networks are all integrated on the Internet. Internet can transparentize the Webpages used by users and their related programs, so that users won't feel the differences. The Web is composed of tens of thousands of Web browsers and servers on the Internet. It processes and analyzes documents and files according to the requirements of users, and connects remote information and network resources in a specific way to extract necessary information and deliver it to users. The Web can use 
data transfer protocols to obtain useful information and content, and interact with browsers using HTTP (Hyper Text Transfer Protocol), which has the following characteristics: support the client/server mode, flexible information transmission, small program size, simple and fast reading, each connection only processes one transmission request, and the server is faster since it's free from the memory function.

The structure of Web is a Browser/Server structure with three layers: presentation layer (WWW browser), function layer (Web server with private network interface), and data service layer (back-end data source). Compared with the traditional network structure, it has the merits of convenient system upgrade, good maintainability, high data security, stable system, open, and flexible.

The Web service system processes the interactions among the three types of entities of service requesters, service providers, and service agents, and performs online operations such as information release, search, and binding. Information release means that the service providers describe, release, and modify the services; information search means that the service requesters find the required services through browse or direct acquisition; and information binding means that the service requesters bind information from the server and perform corresponding settings in the system, thereby remotely obtain the information services provided by the service providers.

\subsection{Data update and migration technology}

In a distance education website system, students and course learners add, update, or delete learning files or data, which will cause changes in the relevant data within the system, and such data updates need to be synchronized to the webpage-end of the teachers so that they can master students' learning progress and figure out the problems existing in the courses. As shown in Figure 1, using the data update and migration technology, the modified data is synchronized to the technical database through the intermediate database for teachers to view the changed data.

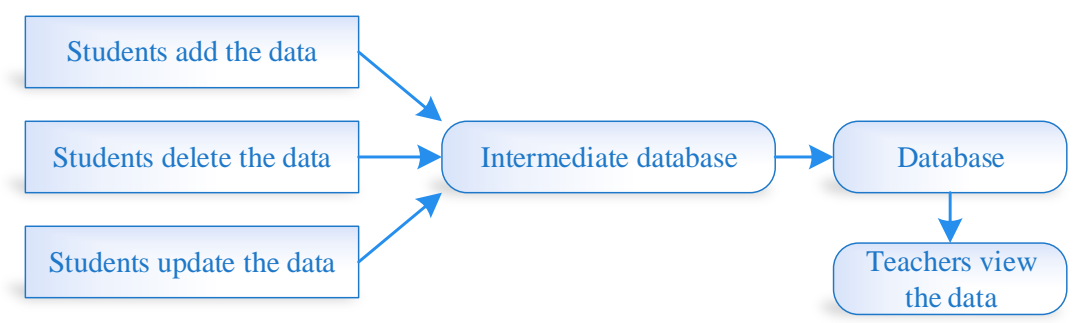

Fig. 1. Data update and migration technology. 


\section{Design of the Distance Education System}

\subsection{Implementation environment}

The design and development of the distance education system of financial management courses includes the whole processes of requirement analysis, webpage design, data analysis, webpage making, and system operation and maintenance, as well as the evaluation and modification according to the requirements and the actual teaching process.

As shown in Figure 2, the design of the distance education system is based on the information needs of the service requesters, that is, evaluate the requirements based on actual teaching situations, and design the system after the evaluation is proved to be qualified; in the system design stage, after the design is completed, it needs to be evaluated as well, if the design does not meet the requirements of the service requesters or there are design flaws, the problematic design is modified, after the design is evaluated to be qualified, it proceeds to the system development stage. Then, after the system development is completed, the final evaluation of the entire system is carried out; if the design goals are met, the service is considered completed, otherwise it needs to be further modified and evaluated.

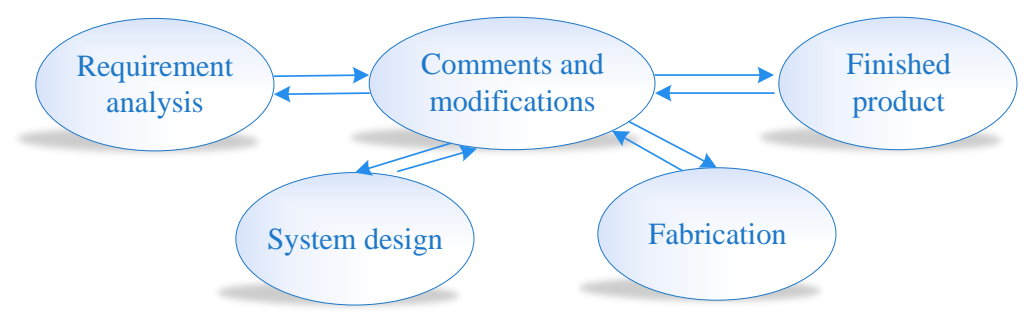

Fig. 2. Design process of the system

A simple client-end mode has been adopted for the operation mode of the distance education system, which has provided a technical guarantee for reducing the costs of system development and operation of schools or other education institutions, and for improving teaching efficiency and updating the information, it has also transformed the traditional classroom teaching mode and facilitated students' course learning and teachers' teaching works. Figure 3 gives a diagram of the operation mode of the distance education system. Even when the teacher is not at school or in the classroom, the teaching could be carried out remotely using the distance education system on the Internet; as for students, when they are at school, they can learn the courses using campus LAN (local area network), when they are not at school, they can learn and communicate online using the public Internet, in this way, students can use their spare time to learn the professional knowledge, and it has reduced the course teaching cost of the schools. 


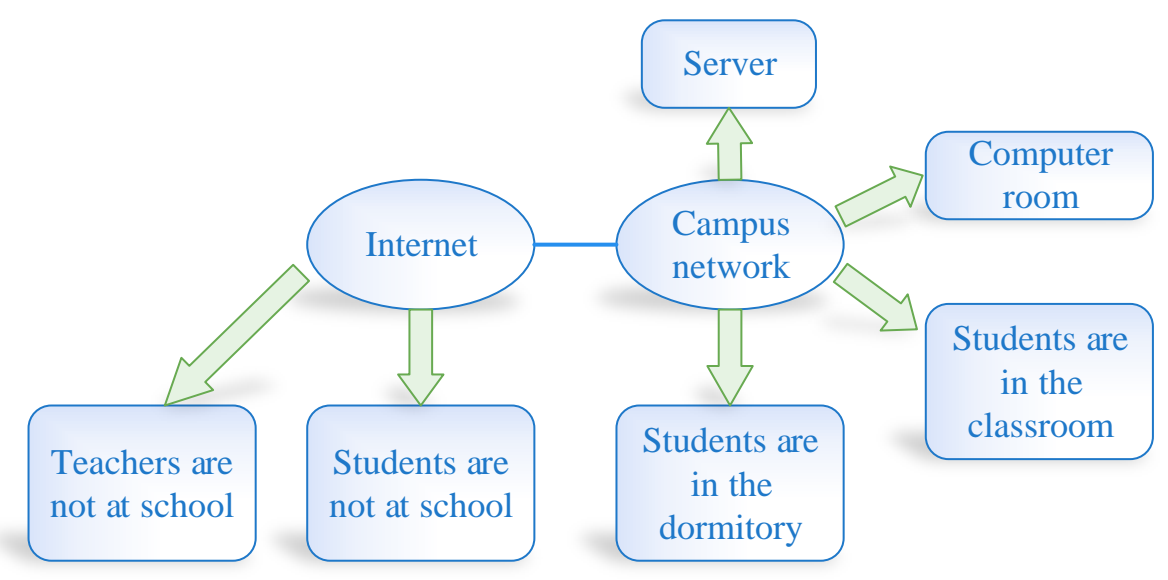

Fig. 3. Operation mode of the system

\subsection{System design}

The program development process of the distance education system of financial management courses is as follows: at first, investigate system requirements and roughly determine the webpages and function interfaces of the system; second, give preliminary design and determine the distance education system's organization structure, management modules, functions, operations, data structure, and post-processing, etc.; third, give detailed system design, adopt dynamic webpages and computer algorithms, determine information structure and algorithm call relationships, and encode and test using computer language; fourth, after the dynamic webpages and the system are completed, realize the interaction of different modules and the real-time data update, test and try the compiled system, after passing the evaluation, the system could be delivered to the schools and education institutions for use. In addition, during the process of use, the system and the webpages need to be optimized and updated per the requirements of the service requesters. 


\subsection{System structure}

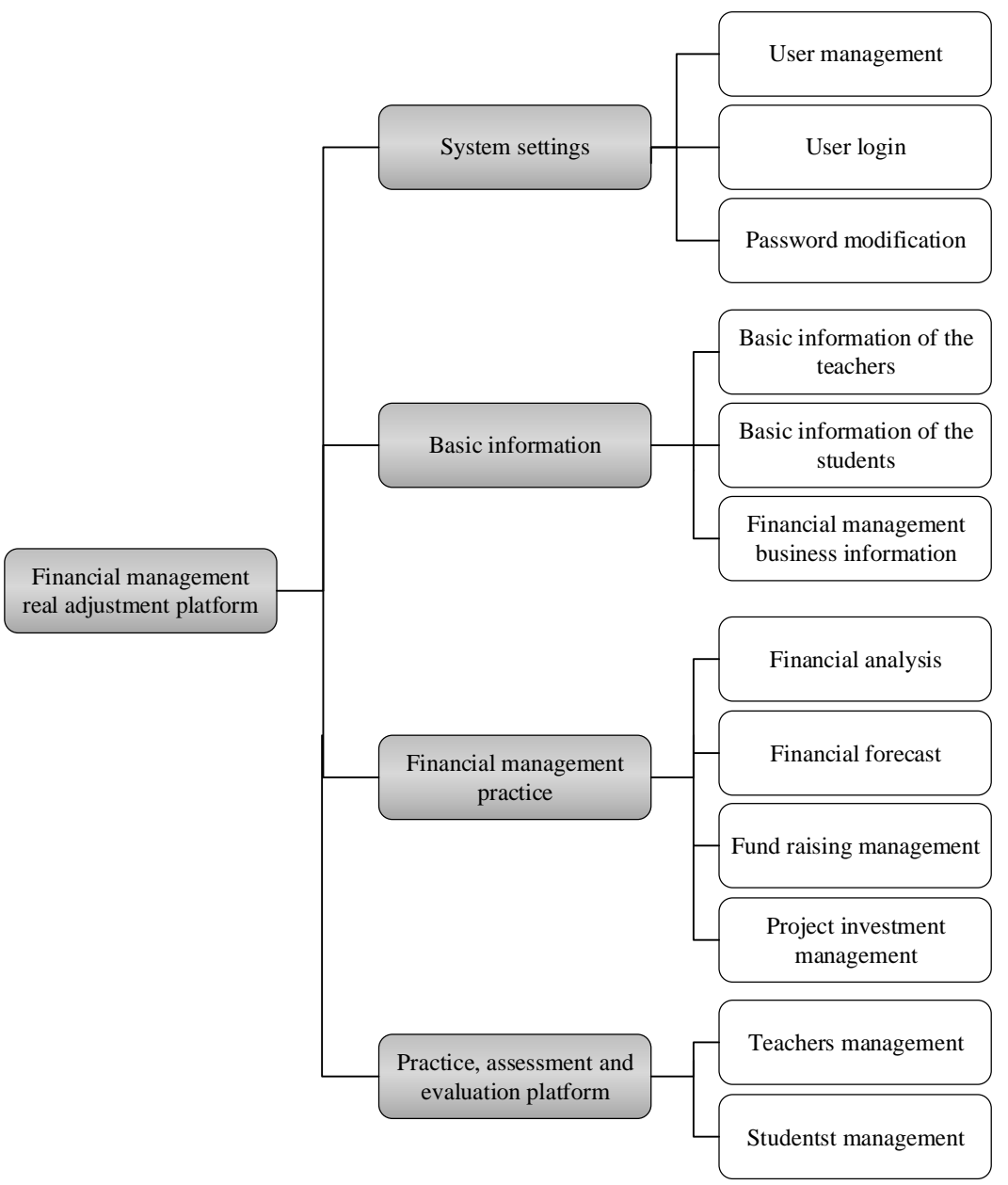

Fig. 4. Structure of the distance education system of financial management courses

According to functions, the proposed system could be divided into a few modules, including basic settings, user information, course teaching, and exercise and exam, as shown in Figure 4. The basic setting module includes functions such as user management, user register, and password management, etc., this module enables student and teacher users to register, fill in information, change password, and input user rights, etc. The user information module contains the basic information of teacher and student users, as well as the information of the financial management courses. The course teaching module includes contents such as financial analysis, financial forecast, fund management, and project investment management, etc., through online courses and question discussion, users can use this module to learn the professional 
knowledge. The exercise and exam module enables students to assess their mastery of the professional knowledge, and it also enables the teachers to know the learning progress of the students. These modules are independent of each other, and interact with each other; the logical relationships between the modules have been considered in the system design and development process.

The exercise module contains various contents such as the basic company information, product introduction, production capacity, organizational structure and personnel, financial status, risk prediction and evaluation, and financial statements, etc. Students can browse these contents via the dynamic webpages, get to know the relevant information of the company, and use professional knowledge to calculate the annual financial management goals that need to be achieved within the calculation period, and information could be provided for subsequent financial management and decision-making works based on the financial analysis results. In addition, learners can use options on the financial management environment page to view relevant information, the specific operation process is shown in Figure 5.

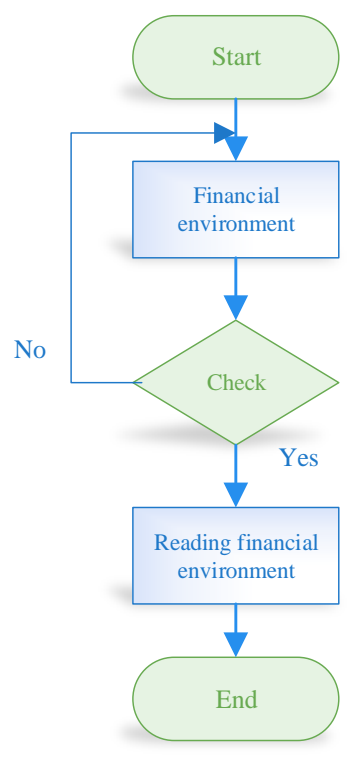

Fig. 5. Process of viewing financial management environment

Financial analysis is an important part of financial management. Its main content is to analyse and summarize the financial management situations of the company combining with the financial information and data of the company in the exercise module. As shown in Figure 6, the financial analysis webpages provide trainees or students with corporate financial data of many years, on this basis, the multiple financial analysis indicators and data are integrated and the evaluation and analysis of these multiple indicators are built on the analysis platforms for students to learn how to use, evaluate, and analyze these indicators, in this way, students can have a comprehensive understanding of the process of financial analysis. 


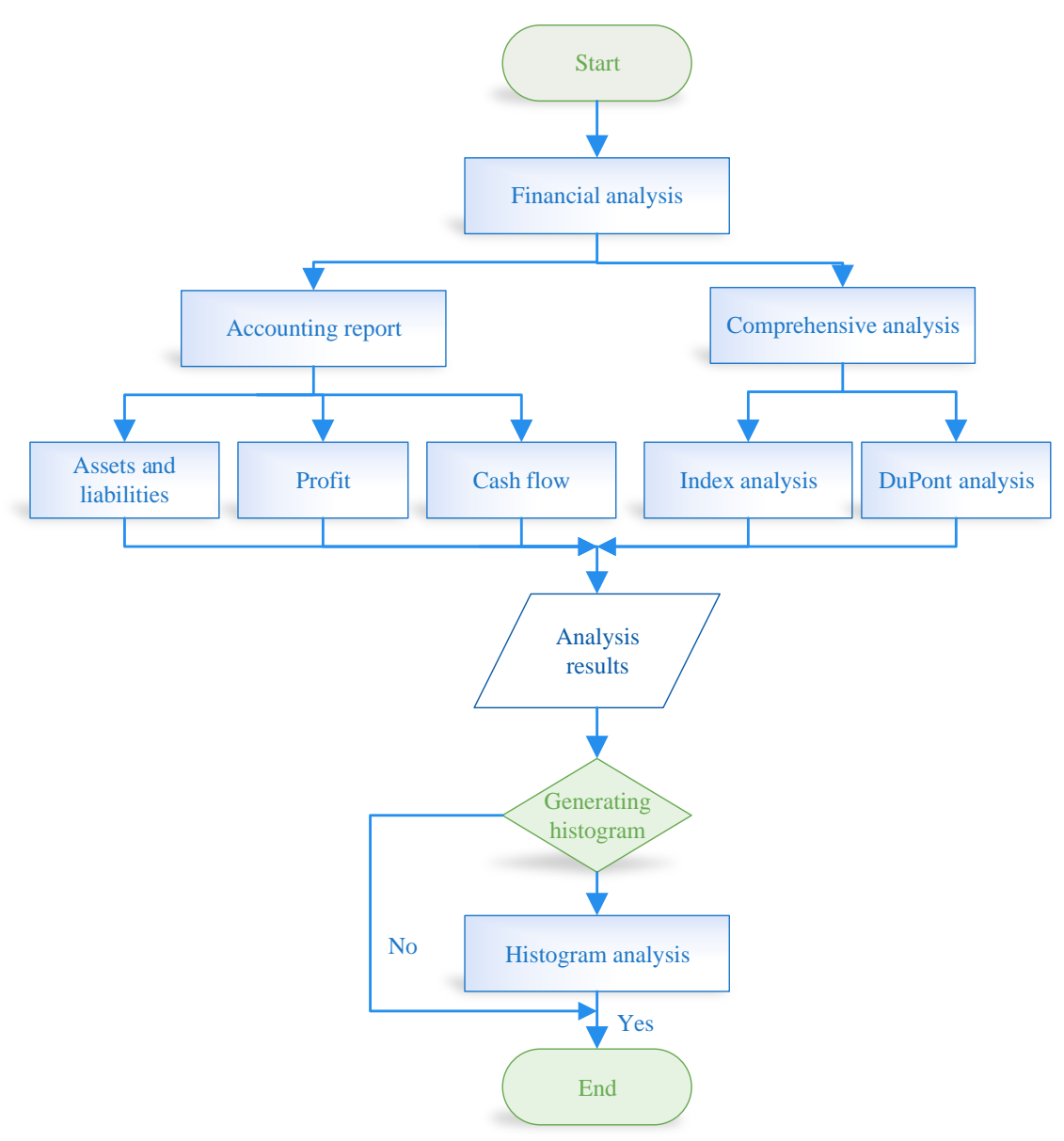

Fig. 6. Process of financial analysis

The financial budget module scientifically evaluates and budgets the future financial activities and results of the company based on related data of the financial activities of the company, and the annual financial requirements and conditions, as shown in Figure 7, the main content of this module is to measure and calculate the economic benefits of the production and management of the company, and offer reliable references for the decision-making of the company; moreover, it can predict the trends of the company's income and expenditure, determine the business objectives, and then prepare plans for the future development of the company. 


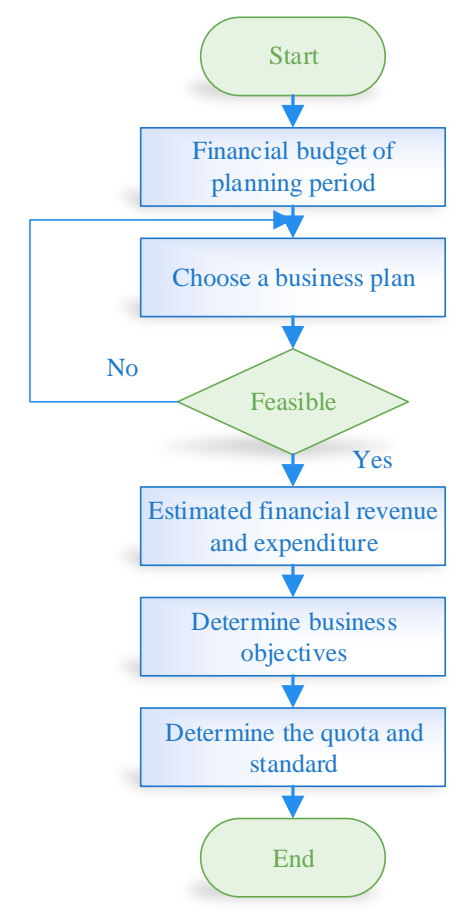

Fig. 7. Process of financial budget

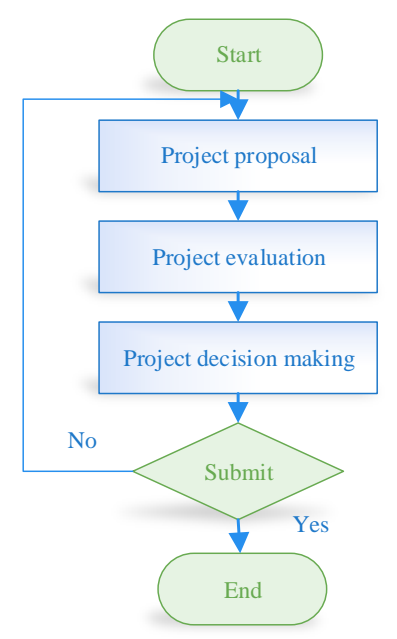

Fig. 8. Process of project investment management

The project investment management is a core content of financial management, it is also the application of the analysis results of above parts. Aiming at other parts of the financial management environment module, students or trainees can propose sev- 
eral project investment plans based on the corporate financial budgets, financial management goals, and fund-raising situations of the company, then, after further analysis, they can make the related decisions, and input the final plans into the dynamic webpages of the financial management system.

\section{Application of the Distance Education System of Financial Management Courses}

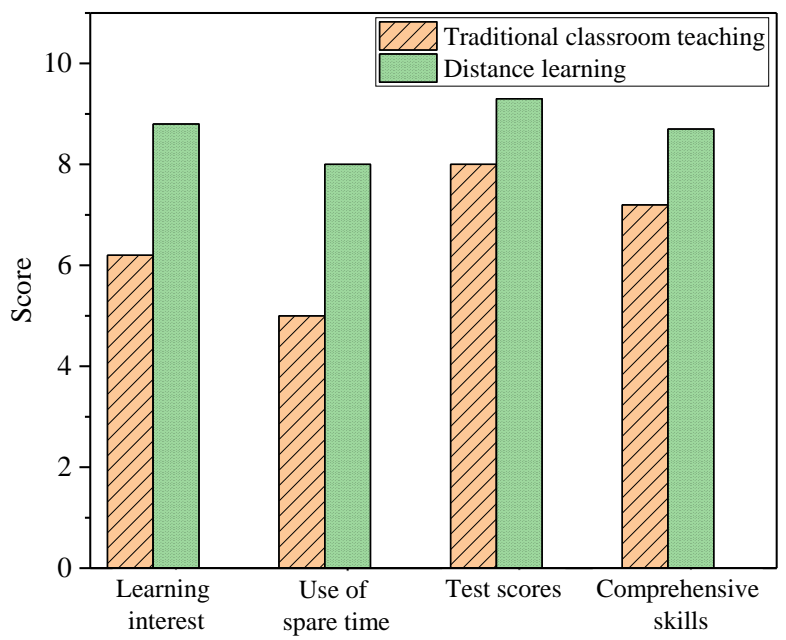

Fig. 9. Comparison of the learning effect of traditional classroom teaching mode and the distance education mode

In order to compare and analyse the impact of online distance education on the teaching of financial management courses, a questionnaire survey was conducted to investigate the learning situations of 100 students/trainees (including their learning interest, free learning time, exam scores, and mastery of professional skills), and the results were counted and analysed. With 10 points as the highest score (best learning effect and full score), and 0 point as the lowest score (worst learning effect), the four survey questions were scored and the results are shown in Figure 9. Compared with traditional classroom teaching method, using distance education system to teach financial management courses can greatly improve the students' professional knowledge learning efficiency, enable them to make full use of their spare time to increase knowledge accumulation and exchange with others about the professional issues, in this way, students' exam results and professional skill mastery levels could be improved. 


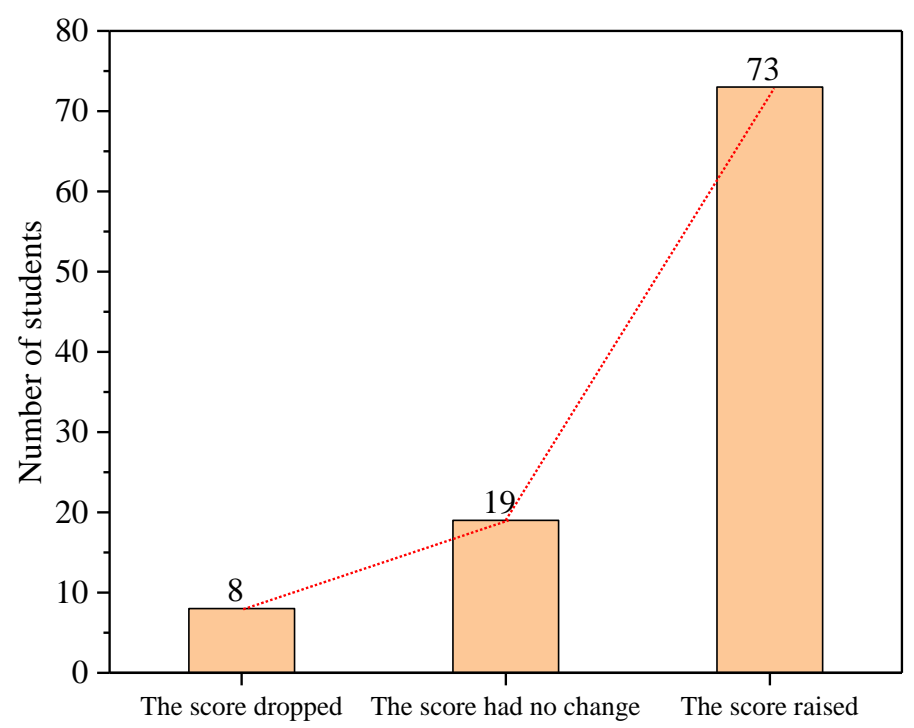

Fig. 10.Statistics of students' exam scores after the distance education mode had been adopted

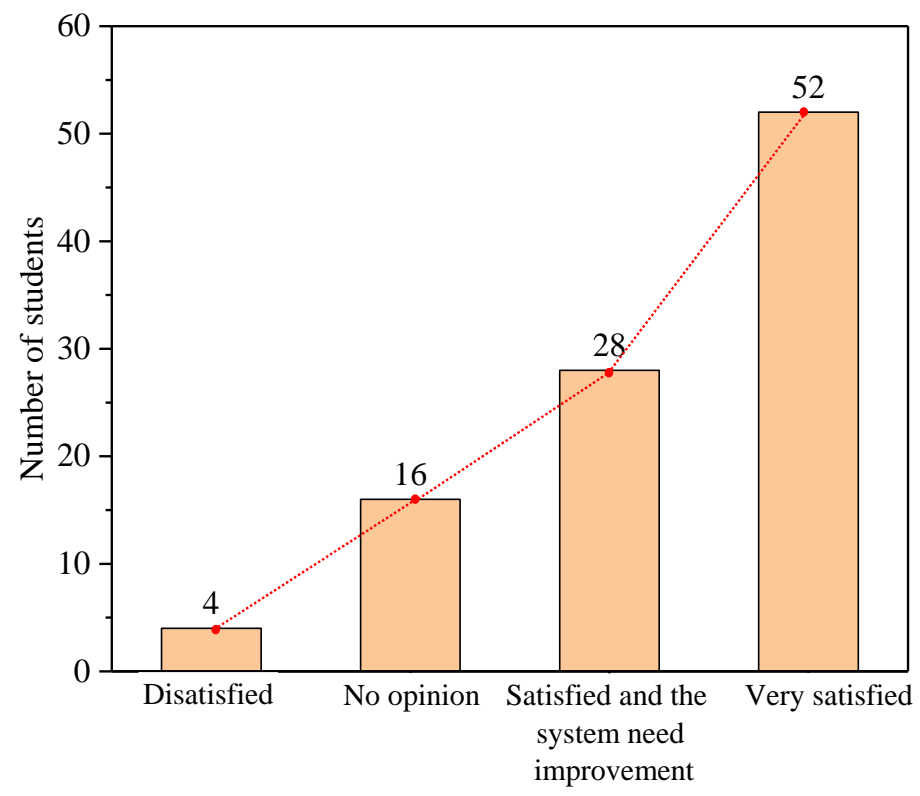

Fig. 11. Survey results on students' satisfaction with the distance education system

After the distance education mode had been adopted, students' exam scores in the financial management courses had been improved, and the situation is shown in Fig- 
ure $10,73 \%$ of the students' professional course scores improved significantly; $19 \%$ of the students' scores didn't change; and $8 \%$ of the students' scores declined, which might be caused by the fact that these students failed to adapt to the new teaching mode in time and their online learning time hadn't been properly arranged. The survey results on the satisfaction of students/trainees with the distance education system and webpages of the financial management courses are shown in Figure 11, only $4 \%$ of the students are not satisfied with the distance education mode; $16 \%$ of students have no opinion on distance education; $28 \%$ of them are satisfied with this teaching mode but think that the distance education system needs to be improved; $52 \%$ of the students are very satisfied with this system. According to these results, we can see that the distance education mode of the financial management courses has been recognized by most students.

\section{Conclusion}

Based on dynamic webpage design and computer programming technologies, this study designed a distance education system for financial management courses, which integrated the teaching resources of the financial management courses into the webpages, the main conclusions of this study are as follows:

1. The distance education system of financial management courses realized online communication and exchange between students and teachers, and provided users with a few functions such as user creation, user right management, and system parameter setting, which is convenient for users to register and use the system.

2. In the proposed system, users can query basic information and search for training cases that are related to financial management; for each module, users can set the information separately, at the same time, the different modules can interact with each other and be used for the online operation and skill training of users.

3. The proposed system embedded real corporate financial management cases and can provide students with actual financial management training programs. In the simulated environment, students can become real corporate decision-makers, and this is very important for cultivating students' financial management skills.

\section{Acknowledgement}

Subject: JRS-2020-1138 2020 Hebei Province Human Resources and Social Security Research Project General Project (The impact of the new crown pneumonia epidemic on the employment of fresh graduates in our province and decision-making suggestions). 


\section{$7 \quad$ References}

[1] Meyer, M. (2004). Corporate real estate and Sarbanes-Oxley: Web-based tools for ensuring compliance and improving financial management. Journal of Corporate Real Estate, 6(1): 83-91. https://doi.org/10.1108/14630010410812252

[2] Cappabianca, D.D. (2002). The Knowledge Management Imperative for USAF Financial Management. The Air Force Controller, January, 7-10.

[3] Spinciu, M. (2017). Financial Management Software and Planning Security. Contemporary Economy Journal, 2(3): 239-243.

[4] Lawson, K.A. (1991). Using a computer-simulation program and a traditional approach to teach pharmacy financial management. American Journal of Pharmaceutical Education, 55(3): 226-35. https://doi.org/10.1016/0741-8329(91)90693-Q

[5] Edwardson, S.R., Pejsa, J. (1993). A computer assisted tutorial for applications of computer spreadsheets in nursing financial management. Computers in nursing, 11(4): 169-175.

[6] Smith, S., Gale, S., Malampy, W. (1995). A Financial Management Approach for Selecting Optimal, Cost-effective Safeguards Upgrades for Computer-and Information-Security Risk Management. Computers \& Security, 1(14): 28-29. https://doi.org/10.1016/01674048(95)97000-Z

[7] Yang, D.Z. (2014). Discussion on the Usage of Computer-assisted Method in Evaluation of Enterprise Financial Management. In Applied Mechanics and Materials, 543: 42354238. https://doi.org/10.4028/www.scientific.net/AMM.543-547.4235

[8] Wang, Y., Sun, C., Guo, Y. (2020). A Multi-Attribute Fuzzy Evaluation Model for the Teaching Quality of Physical Education in Colleges and Its Implementation Strategies, International Journal of Emerging Technologies in Learning, 16(20): 159-172. https://doi.org/10.3991/ijet.v16i02.19725

[9] Abdul-Rahman Al-Malah, D.K., Hamed, S.I., Haider, T.H., ALRikabi, S. (2020). The Interactive Role Using the Mozabook Digital Education Application and its Effect on Enhancing the Performance of eLearning, International Journal of Emerging Technologies in Learning, 15(20): 21-41. https://doi.org/10.3991/ijet.v15i20.17101

[10] Tyupikova, T.V., Samoilov, V.N. (2003). Computer networks for financial activity management, control and statistics of databases of economic administration at the Joint Institute for Nuclear Research. Nuclear Instruments and Methods in Physics Research Section A: Accelerators, Spectrometers, Detectors and Associated Equipment, 502(2-3): 749-751. https://doi.org/10.1016/S0168-9002(03)00565-5

[11] Utomo, M.N.Y., Sudaryanto, M., Saddhono, K. (2020). Tools and strategy for distance learning to respond COVID-19 pandemic in Indonesia. Ingénierie des Systèmes d'Information, 25(3): 383-390. https://doi.org/10.18280/isi.250314

[12] Irungu, J.M. (2002). Headteachers' preparation and support in financial management: A study of public secondary schools in Nakuru municipality, Kenya. Unpublished M. Ed. Thesis, Kenyatta. University, Nairobi.

[13] Sun, Y., Chai, R.Q. (2020). An early-warning model for online learners based on user portrait. Ingénierie des Systèmes d'Information, 25(4): 535-541. https://doi.org/10.18280/ $\underline{\text { isi. } 250418}$

[14] Nagata, K., Nakamura, T., Geurdes, H., Batle, J., Abdalla, S., Farouk, A. (2018). Creating very true quantum algorithms for quantum energy-based computing. International Journal of Theoretical Physics, 57(4): 973-980. https://doi.org/10.1007/s10773-017-3630-1

[15] Mangiero, G.A., Manley, J., Mollica, J.T. (2010). Improving pedagogy through the use of dynamic Excel presentations in financial management courses. American Journal of Business Education (AJBE), 3(1): 91-106. https://doi.org/10.19030/ajbe.v3i1.377 
[16] Batle, J., Ooi, C. R., Farouk, A., Alkhambashi, M. S., Abdalla, S. (2016). Global versus local quantum correlations in the Grover search algorithm. Quantum Information Processing, 15(2): 833-849. https://doi.org/10.1007/s11128-015-1174-y

[17] Xia, W.H. (2013). Research on Financial Management Method of Fixed Assets of Large Enterprise Based on Computer Information Technology. In Applied Mechanics and Materials, 380: 4676-4680. https://doi.org/10.4028/www.scientific.net/AMM.380-384.4676

[18] Hove, M., Wynne, A. (2010). The Experience of Medium-Term Expenditure Framework \& Integrated Financial Management Information System Reforms in Sub-Saharan AfricaWhat is the Balance sheet.

[19] Nagata, K., Nakamura, T., Farouk, A. (2017). Quantum cryptography based on the Deutsch-Jozsa algorithm. International Journal of Theoretical Physics, 56(9): 2887-2897. https://doi.org/10.1007/s10773-017-3456-X

[20] Wang, S., Liu, D., Wang, N., Yuant, Y. (2020). Design and Implementation of an Online Python Teaching Case Library for the Training of Application Oriented, International Journal of Emerging Technologies in Learning, 15(21): 217-230. https://doi.org/10.3991/ ijet.v15i21.18191

[21] Park, S.J., Cho, K.H. (2016). Power-based supervisory control theory of hybrid systems and its application to the analysis of financial crisis. IET Control Theory \& Applications, 10(7): 780-788. https://doi.org/10.1049/iet-cta.2015.1047

\section{Author}

Fang Yu is a lecturer in the Department of Economics and Trade of Shi Jia Zhuang university of applied technology, China. She graduated from the University of Reading in the UK. Her research interests include accounting, financial management, and statistics. She has published four papers and participated in one project.

Article submitted 2021-05-03. Resubmitted 2021-05-29. Final acceptance 2021-06-02. Final version published as submitted by the authors. 